\title{
Alignment and Morphing for the Boundary Curves of Anatomical Organs
}

\author{
Keiko Morita $^{1}$, Atsushi Imiya ${ }^{2}$, Tomoya Sakai $^{3}$, \\ Hidetaka Hontan ${ }^{4}$, and Yoshitaka Masutani ${ }^{5}$ \\ 1 School of Advanced Integration Sciences, Chiba University, Japan, \\ Yayoi-cho 1-33, Inage-ku, Chiba, 263-8522, Japan \\ 2 Institute of Media and Information Technology, Chiba University, Japan, \\ Yayoi-cho 1-33, Inage-ku, Chiba, 263-8522, Japan \\ 3 Department of Computer and Information Science, Nagasaki University, \\ Bunkyo-cho 1-44, Nagasaki, 852-8521, Japan \\ 4 Department of Computer Science, Nagoya Institute of Technology, Gokiso, \\ Showa-ku, Nagoya 466-8555, Aichi, Japan \\ 5 Department of Radiology, The University of Tokyo Hospital, \\ Division of Radiology and Biomedical Engineering, Graduate School of Medicine \\ The University of Tokyo, \\ Hongo 7-3-1, Bunkyo-ku 113-8655, Tokyo, Japan
}

\begin{abstract}
In this paper, we develop a tracking method for the deformable boundary curves of biological organs using variational registration method. We first define the relative distortion of a pair of curves using curvatures of curves. This minimum distortion aligns corresponding points of a pair of curves. Then, we derive the mean of curves as the curve which minimises the total distortion of a collection of shapes. We compute the intermediate boundary curve of a pair of curves as the mean of these curves.
\end{abstract}

\section{Introduction}

Morphing is a fundamental technique in computer graphics to interpolate and generate shapes and objects. In medical application, morphing is used for the description of deformation process of biological organs. This process predicates deformable motion of biological organs in human torso such as beating heart, deformation of lungs during blessing. Follow up analysis of tumors in censer diagnosis tracks and predicates deformation of censer. In this paper, we develop a tracking method for the deformable boundary curves of biological organs using variational registration method. This registration process between images clarifies the difference between images which is used for medical diagnosis. This registration process is mainly achieved by the matching process, which is an established fundamental idea in pattern recognition. In both structure pattern recognition [4, 5] and variational registration [1, 3], the mean shape of a collection of given shapes is interested. 
Shape retrieval categorises and classifies shapes, and finds shapes from portions of shapes. In shape retrieval, the matching of shapes based on the defomorphorism of shapes [8, 9, 16, 19] and descriptor of shape boundary contours [10] are used. In the matching process for discrete shapes, the string edit-distance [4, 6] computed by dynamic programming is a fundamental tool. Moreover, in the matching process of images, the variational registration strategy [1, 2] is a typical tool. Since, in registration of grey-valued images, the deformation is assumed to be relatively small, the point correspondences between the target and reference images are estimated as a local deformation of images [1-3]. For the matching of planar curves, we are required to estimate both alignments and local deformation of curves. In this paper, we separate this problem into alignment estimation [16, 17] in the normalised set of curves and deformation of curves.

\section{Alignment of Curves}

For a pair of planar curves $\boldsymbol{c}(s)=\left(c_{1}(s), c_{2}(s)\right)^{\top}$ and $\overline{\boldsymbol{c}}(s)=\left(\bar{c}_{1}(s), \bar{c}_{2}(s)\right)^{\top}$, whose lengths are $C$ and $\bar{C}$, respectively, assuming $\boldsymbol{c}(s+C)=\boldsymbol{c}(s)$ and $\overline{\boldsymbol{c}}(s+\bar{C})=$ $\overline{\boldsymbol{c}}(s)$, the alignment of curves is obtained as

$$
\operatorname{Align}(c, \bar{c})=\min _{t, \psi} \int_{0}^{C}|\overline{\boldsymbol{c}}(\psi(s)-t)-\boldsymbol{c}(s)|^{2} d s,
$$

where $\psi(\tau)$ is a monotone function from the interval $[0, C]$ to the interval $[0, \bar{C}]$ [13, 14, 16]. The function $\tau=\psi(s)$ and the displacement $t$ define the correspondences of points on a pair of curves $\boldsymbol{c}(s)$ and $\overline{\boldsymbol{c}}(\tau)$.

The dynamic time warping (DTW) is a fundamental procedure to achieve curve alignment employing dynamic programming [13]. The time warping sometimes maps a point on a curve to a relatively long interval of another curve. The derivative dynamic time warping technique (DDTW) 14], which computes alignment of derivative curves, solves this pathological mapping. Therefore, we can also use

$$
\operatorname{Align}(c, \bar{c})=\min _{t, \psi} \int_{0}^{C}|\dot{\overline{\boldsymbol{c}}}(\psi(s)-t)-\dot{\boldsymbol{c}}(s)|^{2} d s,
$$

for the derivative of curves [14] $\dot{\boldsymbol{c}}$ and $\dot{\overline{\boldsymbol{c}}}$.

For a planar curve $S_{i}$, the normal curve $s_{i}$ is the curve whose length are normalised to unity is a normalised curve. For the normal curve $\boldsymbol{x}(s)$ the unit normal vector is $\boldsymbol{n}(s)=(-\sin \theta(s), \cos \theta(s))^{\top}$, if $\dot{\boldsymbol{x}}(s) /|\dot{\boldsymbol{x}}(s)|=(\cos \theta(s), \sin \theta(s))^{\top}$. We call $\theta(s)$ the $p$-expression of the curve. The $p$-expression is invariant for Euclidean motion, that is, for curve $\boldsymbol{x}(s), \boldsymbol{y}(s)=\boldsymbol{x}(s)+\boldsymbol{a}$ derives the same $p$-expression.

We define the log measure between two normal curves as

$$
H\left(\theta_{1}, \theta_{2}\right)=\int_{0}^{1}\left|\ln \frac{\exp \left(i \theta_{1}\left(s-t_{1}\right)\right)}{\exp \left(i \theta_{2}\left(s-t_{2}\right)\right)}\right|^{2} d s,
$$


using $p$-expression of each curve of a pair. Using $p$-expression of each curve of a pair, the $p$-distance of a pair of simple polygonal curve $S_{i}$ and $S_{j}$ is defined by

$$
D\left(S_{i}, S_{j}\right)^{2}=\min H\left(\theta_{i}, \theta_{j}\right)=\min _{t} \int_{0}^{1}\left|\theta_{i}(s-t)-\theta_{j}(s)\right|^{2} d s .
$$

where $\theta_{i}$ and $\theta_{j}$ are the $p$-expressions of the normalised curve $s_{i}$ and $s_{j}$ of $S_{i}$ and $S_{j}$, respectively. Then, setting

$$
t_{i j}=\arg \left(\min _{t}\left(\int_{0}^{1}\left|\theta_{i}\left(s-t_{i j}\right)-\theta_{j}(s)\right|^{2} d s\right),\right.
$$

we define the alignment of $s_{i}$ and $s_{j}$ as $\theta_{i}\left(s-t_{i j}\right)$ and $\theta_{j}(s)$.

The $p$-expression $\theta(k)$ of a normalised polygonal curve, whose vertices are $\left\{\boldsymbol{x}_{k}\right\}_{k=1}^{n}$, is computed as

$$
\frac{\boldsymbol{x}_{k}-\boldsymbol{x}_{k-1}}{\left|\boldsymbol{x}_{k}-\boldsymbol{x}_{k-1}\right|}=(\cos \theta(k), \sin \theta(k))^{\top}
$$

Furthermore, setting $\theta_{i}^{k}=\theta_{i}(k \Delta)$, the distance between a pair of normalised curves is approximately computed by

$$
d_{i j} \approx \min _{p} \sum_{k=1}^{m}\left|\theta_{i}^{k}-\theta_{j}^{k-p}\right|^{2}
$$

for an appropriately large $m$ such that $\Delta m=1$.

Next, we define the mean $\phi_{i j}(s)$ of a pair of $p$-expressions $\theta_{i}(s)$ and $\theta_{j}(s)$ as the minimiser of the functional

$$
\begin{aligned}
J\left(\phi_{i}, \phi_{j}, \phi_{i j}, t_{i}, t_{j}\right)= & \int_{0}^{1}\left\{\left|\left(\theta_{i}\left(s-t_{i}\right)-\phi_{i}(s)\right)-\phi_{i j}(s)\right|^{2}\right. \\
& +\left|\left(\theta_{j}\left(s-t_{2}\right)-\phi_{j}(s)\right)-\phi_{i j}(s)\right|^{2} \\
& \left.+\lambda\left|\dot{\theta}_{i j}(s)\right|^{2}+\mu\left|\dot{\phi}_{i}(s)\right|^{2}+\mu\left|\dot{\phi}_{j}(s)\right|^{2}\right\} d s .
\end{aligned}
$$

Equation (8) is converted to the problem,

$$
\begin{aligned}
J_{2}\left(\phi_{i j}\right)= & \int_{0}^{1}\left\{\left|\left(\theta_{i}\left(s-t_{i j}\right)-\phi_{i}(s)-\phi_{i j}(s)\right)\right|^{2}\right. \\
& +\mid\left(\theta_{j}(s)-\phi_{j}(s)-\left.\phi_{i j}(s)\right|^{2}\right. \\
& \left.+\lambda\left|\dot{\phi}_{i j}(s)\right|^{2}+\mu\left|\dot{\phi}_{i}(s)\right|^{2}+\mu\left|\dot{\phi}_{j}(s)\right|^{2}\right\} d s
\end{aligned}
$$

since $t_{i j}$ aligns a pair of $p$-expressions, for a generalisation of eq. (9), the initial points of a collection of curves are required to be aligned. 


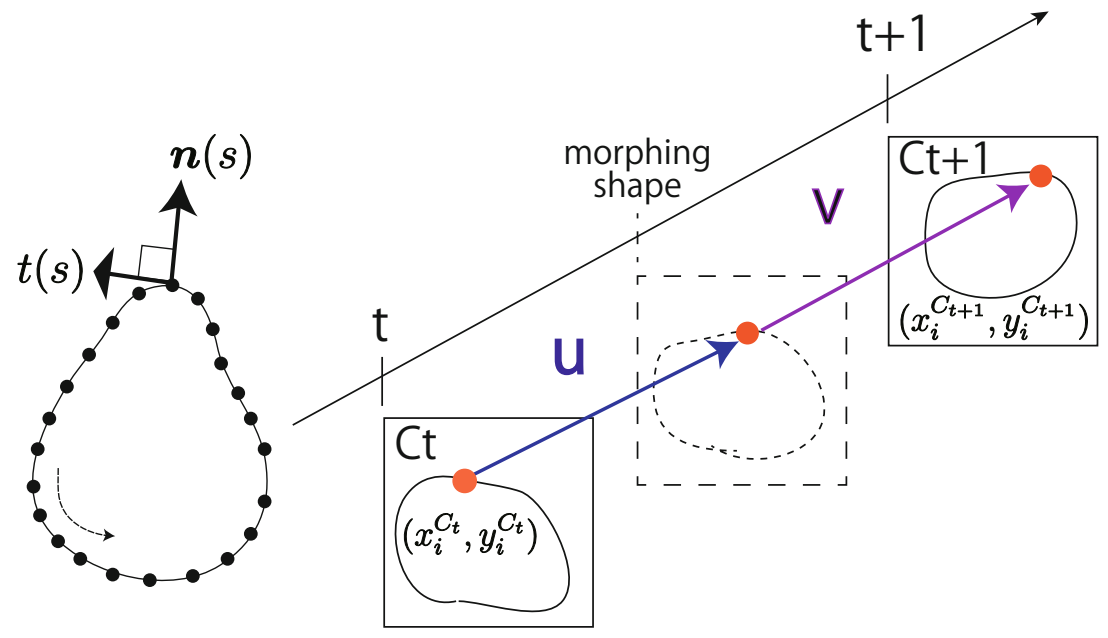

(a)

(b)

Fig. 1. Geometric property of the image boundary of curve. (a) Configuration of the normal and tangent vectors on a planar simple curve. (b) Morphing of temporal curves.

\section{The Mean of Polygonal Curves}

Assuming that the correspondence of the vertices of curves $S_{i}$ and $S_{j}$ in a collection of curves $\left\{S_{\alpha}\right\}_{\alpha}^{n}$ are established by minimising eq. (3), we define the distance between a pair of polygonal curves $S_{i}=\left\{\boldsymbol{f}_{i k}\right\}_{k=1}^{n}$ and $S_{j}=\left\{\boldsymbol{f}_{j k}\right\}_{k=1}^{n}$ where $\boldsymbol{f}_{\cdot k}=\left(x_{\cdot k}, y_{\cdot k}\right)^{\top}$ is the vertex of the curve $S$. with the condition $\boldsymbol{f}_{\cdot m+k}=\boldsymbol{f}_{\cdot k}$, as

$$
\begin{aligned}
d\left(S_{i}, S_{j}\right) & =\min _{\boldsymbol{u}_{k}^{i j}}\left\{\sum_{k=1}^{m}\left\{\left(\boldsymbol{f}_{i k}-\boldsymbol{u}_{k}^{i j}\right)-\boldsymbol{f}_{j k}\right\}^{2}+\mu \sum_{k=1}^{m}\left(\bar{\nabla} \boldsymbol{u}_{k}^{i j}\right)^{2}\right\} \\
& =\min _{\boldsymbol{u}_{k}^{i j}}\left\{\sum_{k=1}^{m}\left\{\boldsymbol{f}_{i k}-\left(\boldsymbol{f}_{j k}-\boldsymbol{u}_{k}^{j i}\right)\right\}^{2}+\mu \sum_{k=1}^{m}\left(\bar{\nabla} \boldsymbol{u}_{k}^{j i}\right)^{2}\right\} \\
& =d\left(S_{j}, S_{i}\right)
\end{aligned}
$$

where $\boldsymbol{u}_{k}^{i j}=-\boldsymbol{u}_{k}^{j i}$ is the displacement between $\boldsymbol{f}_{i k}$ and $\boldsymbol{f}_{j k}$ and $\bar{\nabla}$ stands for the discrete differential operation along a polygonal curve 1 .

Definition 1. Setting $\boldsymbol{u}_{k i}$ to be deformation of the vertex $\boldsymbol{f}_{k i}$ of the shape $S_{i}$, the vertices $\boldsymbol{g}$ of the mean curve $S$ of $S_{i}$ and $S_{j}$ is the minimiser of the discrete variational problem

$$
J\left(S_{i}\left(\boldsymbol{f}_{i}\right), S(\boldsymbol{g}), S_{j}\left(\boldsymbol{f}_{j}\right)\right)=d\left(S_{i}, S\right)+d\left(S, S_{j}\right)+\lambda P(S)
$$

$\overline{1 \bar{\nabla} \boldsymbol{g}_{k}=\boldsymbol{g}_{k+\frac{1}{2}}}-\boldsymbol{g}_{k-\frac{1}{2}}$ and $\bar{\nabla}^{2} \boldsymbol{g}_{k}=\frac{1}{2}\left(\boldsymbol{g}_{k+1}-2 \boldsymbol{g}_{k}+\boldsymbol{g}_{k-1}\right)$. 
where

$$
\begin{aligned}
d\left(S_{i}, S\right) & =\sum_{k=1}^{n}\left\{\left(f_{i k}-\boldsymbol{u}_{i k}\right)-\boldsymbol{g}_{k}\right\}^{2}+\left.\sum_{k=1}^{n}|\bar{\nabla}| \boldsymbol{u}_{i k}\right|^{2} \\
d\left(S, S_{j}\right) & =\sum_{k=1}^{n}\left\{\left(f_{j k}-\boldsymbol{u}_{j k}\right)-\boldsymbol{g}_{k}\right\}^{2}+\left.\sum_{k=1}^{n}|\bar{\nabla}| \boldsymbol{u}_{j k}\right|^{2}, \\
P(S) & =\sum_{k=1}^{n}\left|\bar{\nabla} \boldsymbol{g}_{k}\right|^{2} .
\end{aligned}
$$

Setting

$$
\boldsymbol{D}=\frac{1}{2}\left(\begin{array}{cccccc}
-2 & 1 & 0 & \cdots & 0 & 1 \\
1 & -2 & 1 & 0 & \cdots & 0 \\
\vdots & & & & & \\
1 & 0 & \cdots & 0 & 1 & -2
\end{array}\right), \boldsymbol{A}=\left(\begin{array}{cc}
\boldsymbol{I}_{2} & \boldsymbol{e} \\
\boldsymbol{e}^{\top} & m
\end{array}\right), \boldsymbol{M}=\operatorname{Diag}(\underbrace{\mu, \mu}, \lambda) .
$$

where $\boldsymbol{e}=(1,1)^{\top}$, we have the Euler-Lagrange equation of eq. (13)

$$
\left(\boldsymbol{I}_{m+1} \otimes \boldsymbol{D}\right) \boldsymbol{s}=\left(\boldsymbol{M}^{-1} \boldsymbol{A} \otimes \boldsymbol{I}_{2}\right) \boldsymbol{s}-\left(\boldsymbol{M}^{-1} \otimes \boldsymbol{I}_{n}\right) \boldsymbol{c},
$$

where $\boldsymbol{I}_{k}$ is the $k \times k$ identity matrix and

$$
\boldsymbol{s}=\left(\boldsymbol{u}_{i}^{\top}, \boldsymbol{u}_{j}^{\top}, \boldsymbol{g}^{\top}\right)^{\top}, \boldsymbol{c}=\left(\boldsymbol{f}_{i}^{\top}, \boldsymbol{f}_{j}^{\top}, \boldsymbol{f}_{i}^{\top}+\boldsymbol{f}_{j}^{\top}\right)^{\top} .
$$

Rewriting eq. (14) as

$$
B s=K s-c,
$$

the semi-implicit discretisation of the diffusion equation

$$
\frac{\partial s}{\partial t}=\boldsymbol{B} \boldsymbol{s}-\boldsymbol{K} \boldsymbol{s}+\boldsymbol{c}
$$

derives the iteration form

$$
(\boldsymbol{I}+\tau \boldsymbol{K}) \boldsymbol{s}^{(k+1)}=(\boldsymbol{I}+\tau \boldsymbol{B}) \boldsymbol{s}^{(k)}+\tau \boldsymbol{c} .
$$

This iteration form implies that

$$
\boldsymbol{g}=(\underbrace{\mathbf{0}_{n}, \cdots, \mathbf{0}_{n}}_{m}, \boldsymbol{I}_{n}) \boldsymbol{s}^{(\infty)}, \boldsymbol{s}^{(\infty)}=\lim _{k \rightarrow \infty} \boldsymbol{s}^{(k)} .
$$

Setting $\boldsymbol{u}_{t i}$ and $\boldsymbol{u}_{(t+1) i}$ to be deformations from the vertices $\boldsymbol{f}_{t i}$ of the mean curve $S_{t+\frac{1}{2}}$ to vertices $\boldsymbol{f}_{t i}$ and $\boldsymbol{f}_{(t+1) i}$ of $S_{t}$ and $S_{t+1}$, respectively, we define an interframe curve. Fig. 2 shows morphing and tracking of a temporal curve sequence using variational mean curve. 
Definition 2. The vertices $\boldsymbol{f}_{t+\frac{1}{2}}$ of the intermediate shape $S_{t+\frac{1}{2}}$ is the minimiser of the discrete variational problem

$$
J\left(S_{t}\left(\boldsymbol{f}_{t}\right), S_{t+\frac{1}{2}}\left(\boldsymbol{f}_{t+\frac{1}{2}}\right), S_{t+1}\left(\boldsymbol{f}_{t+1}\right)\right)=D\left(S_{t}, S_{t+\frac{1}{2}}\right)+D\left(S_{t+\frac{1}{2}}, S_{t+1}\right)+\lambda P\left(S_{t+\frac{1}{2}}\right)
$$

where

$$
\begin{aligned}
D\left(S_{t}, S_{t+\frac{1}{2}}\right) & =\sum_{k=1}^{n}\left\{\left(f_{i k}-\boldsymbol{u}_{i k}\right)-\boldsymbol{g}_{k}\right\}^{2}+\left.\sum_{k=1}^{n}|\bar{\nabla}| \boldsymbol{u}_{i k}\right|^{2} \\
D\left(S_{t+\frac{1}{2}}, S_{t+1}\right) & =\sum_{k=1}^{n}\left\{\left(f_{j k}-\boldsymbol{u}_{j k}\right)-\boldsymbol{g}_{k}\right\}^{2}+\left.\sum_{k=1}^{n}|\bar{\nabla}| \boldsymbol{u}_{j k}\right|^{2} \\
P\left(S_{t+\frac{1}{2}}\right) & =\sum_{k=1}^{n}\left|\bar{\nabla} \boldsymbol{g}_{k}\right|^{2} .
\end{aligned}
$$

\section{Numerical Examples}

Fig. 2 shows Images of the boundary curves of tumors observed in the years, 1996, 19980, 2000, 2002, and 2004.

Fig. 3 comparative results between the means and the linear averages of the years $Y . Y^{*}$, and $Y^{\diamond}$ express the images of the year $Y$ computed by our method and linear average of corresponding vertices of the curve. In Fig. 4, we have evaluated the distances $D\left(Y, Y^{*}\right)$ and $D\left(Y, Y^{\diamond}\right)$. These results show our method derives smooth intermediate curves, by computing the alignment of corresponding vertices and by minimising the relative distortion of curves.

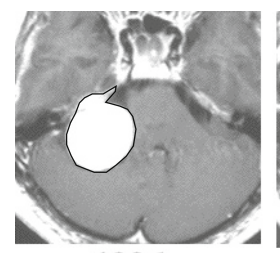

1996

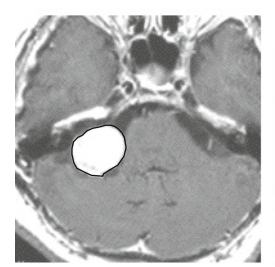

2000

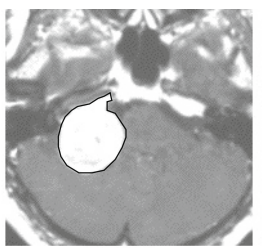

1997

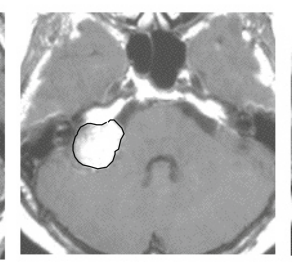

2002

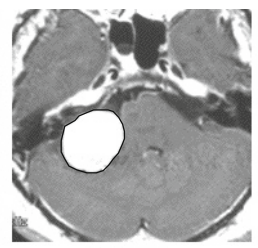

1998

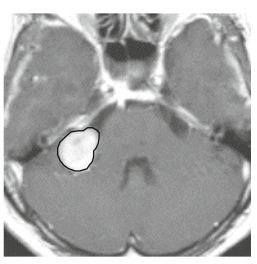

2004

Fig. 2. Images of the boundary curves of tumors. The boundary curves observed in the years, 1996, 19980, 2000, 2002, and 2004. 


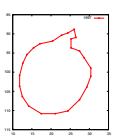

(a) 1997

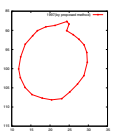

(e) $1997^{*}$

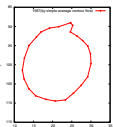

(i) $1997^{\diamond}$

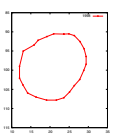

(b) 1998

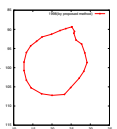

(f) $1998^{*}$

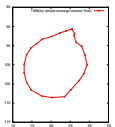

(j) $1998^{\diamond}$

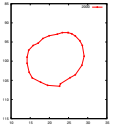

(c) 2000

(d) 2002

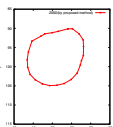

(g) $2000^{*}$

(h) $2002^{*}$

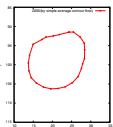

(k) $2000^{\diamond}$
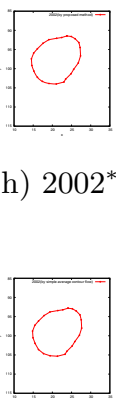

(1) $2002^{\diamond}$

Fig. 3. Performance evaluation. from top to bottom the original images, the means, and the linear averages of the years $Y . Y^{*}$, and $Y^{\diamond}$ express the images of the year $Y$ computed by our method and linear average of corresponding vertices of the curve.

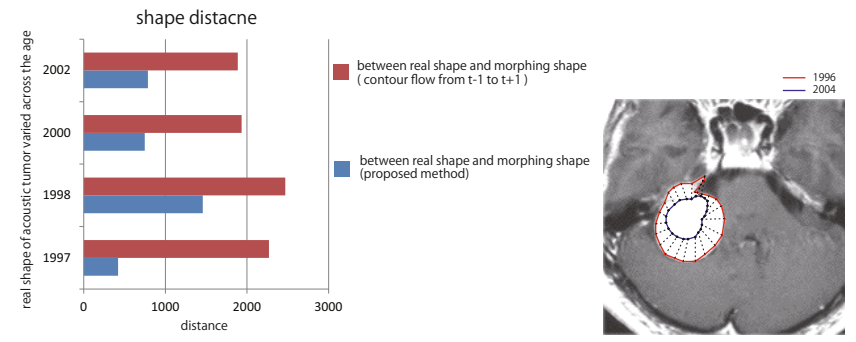

Fig. 4. Evaluation. (a) For the results Fig. 3, we have evaluated the distances $D\left(Y, Y^{*}\right)$ and $D\left(Y, Y^{\diamond}\right)$. (b) Tracking of corresponding vertices between 1996 data and 2004 data.

Fig[5] shows shape morphing results. From the boundary curves observe by the years, 1996, 1998, 2000, 2002, and 2004, the boundary curves of the years $1996+\frac{1}{2}, 1997+\frac{1}{2}, 1998+1,2000+1$, and $2002+1$ are computed. Fig. 4 (b) shows the result for tracking of corresponding vertices between 1996 data and 2004 data. The results show that our method estimates intermediate tumor shapes during the therapy. 


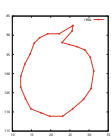

(a) 1996

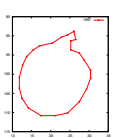

(b) 1997

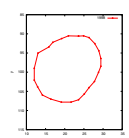

(c) 1998

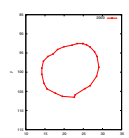

(d) 2000

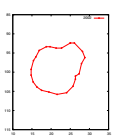

(e) 2002

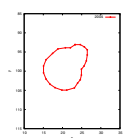

(f) 2004

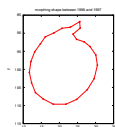

(g)

$1996+\frac{1}{2}$

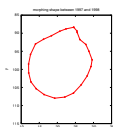

(h)

$1997+\frac{1}{2}$

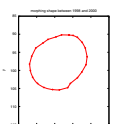

(i) $1998+1$

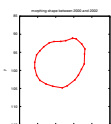

(j) $2000+1$

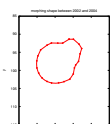

(k)

$2002+1$

Fig. 5. Shape morphing results. from the boundary curves observe by the years, 1996, 19980, 2000, 2002, and 2004, the boundary curves of the years $1996+\frac{1}{2}, 1997+\frac{1}{2}$, $1898+1,2000+1$, and $2002+1$ are computed.

\section{Conclusions}

We first define the relative distortion of a pair of curves using curvatures of curves. This minimum distortion aligns corresponding points of a pair of curves. Then, we derive the mean of curves as the curve which minimises the total distortion of a collection of shapes. We compute the intermediate boundary curve of a pair of curves as the mean of these curves.

Our method automatically detects and tracks corresponding vertices of the temporal-deformation curves.

This research was supported by "Computational anatomy for computer-aided diagnosis and therapy: Frontiers of medical image sciences" funded by the Grantin-Aid for Scientific Research on Innovative Areas, MEXT, Japan, the Grants-inAid for Scientific Research funded by Japan Society of the Promotion of Sciences and the Grant-in-Aid for Young Scientists (A), NEXT, Japan.

\section{References}

1. Hill, D.L.G., et al.: Medical image registration. Phys. Med. Biol. 46, R1-R45 (2001)

2. Fischer, B., Modersitzki, J.: Ill-posed medicine- an introduction to image registration. Inverse Problem 24, 1-17 (2008)

3. Rumpf, M., Wirth, B.: A nonlinear elastic shape averaging approach. SIAM Journal on Imaging Sciences 2, 800-833 (2009)

4. Sebastian, T.B., Klein, P.N., Kimia, B.B.: On aligning curves. IEEE Trans. PAMI 25, 116-125 (2003)

5. Baeza-Yates, R., Valiente, G.: An image similarity measure based on graph matching. In: Proc. 7th Int. Symp. String Processing and Information Retrieval, pp. 8-38 (2000) 
6. Riesen, K., Bunke, H.: Approximate graph edit distance computation by mean s of bipartite graph matching. Image and Vision Computing 27, 950-959 (2009)

7. Mémoli, F.: Gromov-Hausdorff distances in Euclidean spaces. In: NORDIA-CVPR (2008)

8. Arrate, F., Tilak Ratnanather, J., Younes, L.: Diffeomorphic active contours. SIAM J. Imaging Sciences 3, 176-198 (2010)

9. Grigorescu, C., Petkov, N.: Distance sets for shape filters and shape recognition. IEEE Trans. IP 12, 1274-1286 (2003)

10. Tănase, M., Veltkamp, R.C., Haverkort, H.J.: Multiple Polyline to Polygon Matching. In: Deng, X., Du, D.-Z. (eds.) ISAAC 2005. LNCS, vol. 3827, pp. 60-70. Springer, Heidelberg (2005)

11. Arkin, E.M., Chew, L.P., Huttenlocher, D.P., Kedem, K., Mitchell, J.S.B.: An efficiently computable metric for comparing polygonal shapes. IEEE Trans. PAMI 13, 209-216 (1991)

12. Stegmann, M.B., Gomez, D.D.: A brief introduction to statistical shape analysis. Informatics and Mathematical Modelling, Technical University of Denmark (2002), http://www2.imm.dtu.dk/pubdb/p.php?403

13. Müller, M.: In: Information Retrieval for Music and Motion, ch. 4. Springer (2007)

14. Keogh, E.J., Pazzani, M.J.: Derivative dynamic time warping. In: First SIAM International Conference on Data Mining, SDM 2001 (2001), http://www.cs.ucr.edu/ eamonn/

15. Srivastava, A., Joshi, S., Mio, W., Liu, X.: Statistical shape analysis: Clustering, learning, and testing. IEEE Trans. PAMI 27, 590-602 (2005)

16. Sebastian, T.B., Klein, P.N., Kimia, B.B.: On aligning curves. PAMI 25, 116-125 (2003)

17. Marques, J.S., Abrantes, A.J.: Shape alignment? optimal initial point and pose estimation. Pattern Recognition Letters 18 (1997)

18. Kass, M., Witkin, A., Terzopoulos, D.: Snakes: Active contour models. IJCV 1, 321-331 (1988)

19. Sharon, E., Mumford, D.: 2D-shape analysis using conformal mapping. IJCV 70, 55-75 (2006)

20. Mumford, D., Shah, J.: Boundary detection by minimizing functionals. In: Proc. CVPR 1985, pp. 22-26 (1985)

21. Mumford, D., Shah, J.: Optimal approximations by piecewise smooth functions and associated variational problems. Comm. on Pure and Applied Math. bXLII, 577-684 (1989) 